\section{Rui Wang \\ Wei Hua \\ Zilong Dong \\ Qunsheng Peng \\ Hujun Bao}

\title{
Synthesizing trees by plantons
}

Published online: 31 March 2006 (C) Springer-Verlag 2006
R. Wang · W. Hua (®) $\cdot$ Z. Dong ·

Q. Peng · H. Bao

State Key Lab of CAD\&CG, Zhejiang

University, P.R. China

\{rwang, huawei, zldong, peng,

bao\}@cad.zju.edu.cn

\begin{abstract}
In this paper, we present a two-level statistical model for characterizing the stochastic and specific nature of trees. At the low level, we define plantons, which are a group of similar organs, to depict tree organ details statistically. At the high level, a set of transitions between plantons is provided to describe the stochastic distribution of organs.

Based on such a tree model, we propose a novel tree modeling approach, synthesizing trees by plantons, which are extracted from tree samples. All tree samples are captured from the real world. We have designed a maximum likelihood estimation algorithm to acquire the
\end{abstract}

two-level statistical tree model from single samples or multi- samples. Experimental results show that our new model is capable of synthesizing new trees with similar, yet visually different shapes.

Keywords Tree modeling - Modeling from samples $\cdot$ Markov model . Image-based modeling

\section{Introduction}

In many applications such as animation production, video game and flight simulation, realistic trees add significant realism to computer-generated scenes. Nevertheless, modeling trees with high fidelity has been an interesting but difficult problem.

In general, ordinary 3D modeling approaches are not efficient for generating realistic trees due to the trees' vast morphology and complex structure. Since Ulam used the cellular automata to simulate branching patterns in 1962 [36], many tree modeling approaches have been developed. Most of them make use of biological knowledge to either build a parametric model with a few geometric parameters $[31,38]$ or to develop a set of rules to construct trees procedurally $[19,24-27]$ or interactively $[2,18,38]$. These methods are capable of generating various kinds of trees; however, the results heavily rely on the user's biological knowledge and modeling experience. Another modeling technique is based on an inverse-procedure that recovers tree models from photographs $[29,33,35]$. Nevertheless, these solutions aim at resembling trees on photographs rather than at retrieving the botanical principles that can be used to generate new trees.

In this paper, we propose a novel tree modeling approach, efficiently synthesizing trees based on a set of tree samples captured from the real world. We exploit the observation that a tree can be regarded as a composition of some repetitive organs. Moreover, the similarities of organs exist not only within one tree but also across trees of the same species.

Inspired by recent biological research progress [5, 12], we propose a two-level statistical model to represent trees. At the low level, repetitive organs of similar shapes and branching structure are clustered. Such an organ cluster 
is called a planton, which is analogous to a texton [15] in the texture image. For each planton, statistical distributions are used to describe details of the organ. At the high level, we assume that the distribution of these clustered organs in a tree, or namely the transitions between plantons, follows the first-order semi-Markov dynamics, which is represented by a transition matrix. With our tree model, the process of modeling new trees is similar to that of synthesizing texture images based on texture sample. However, while texture images are assumed as a two-dimensional spatial distribution, in our approach trees are assumed as a tree-structured temporal distribution.

To capture more faithful tree samples, we use laser scanning and image-based modeling techniques. Unlike previous methods that attempt to recover a complete tree directly, under the statistical assumption, in our model only a portion of a real world tree needs to be recovered, which greatly reduces the cost of the acquisition and pre-processing. Then, a maximum likelihood estimation algorithm is developed to extract the best plantons and their transitions from samples. Once these plantons and their transitions are learnt, they are used to synthesize new trees with statistically similar, yet visually different shapes.

\section{Related work}

Tree modeling. Many models and approaches have been proposed to represent and model trees. Ulam [36] employed the concept of cellular automata to build the first tree model. Then Greene made an extension introducing a voxel space [11]. Later, deterministic rules and parametric models were used by Honda [14] to describe trees. Such a parametric branching structure and botanical rules were also used by Aono et al. [10] and Weber et al. [38]. In contrast to deterministic models, stochastic models are proposed in $[8,31,37]$. In these methods, tree structures in terms of probabilities are of one-level and all stochastic parameters are specified manually or artificially, not acquired from real world data. Fractals [7,21,23] and rewrite systems $[19,26]$ use a set of rules and a rewriting mechanism to describe the structure and growth of a plant. To enhance the uncontrollable writing rules, more elaborate L-systems were proposed in succession [25, 27]. Other modeling methods, particle systems [30], spline skeletons [13], implicit visualization and inverse modeling technique $[9,10]$ have also been proposed. Recently, some software, which provides an interactive environment to lessen the efforts of designing the model or the rewriting rules, has been developed [2, 18, 38]. Unlike the above approaches, our new method uses captured samples for modeling and it does not require much biological knowledge or modeling experience on the part of the user.
Capturing real-world trees. Recently, several imagebased modeling approaches have been developed to capture trees from the real world. Tatsumi [33] and Shlyakhter [35] employed plant silhouettes on multi-view images to generate a volume representation of a tree, which is used to guide interactively or procedurally $3 \mathrm{D}$ tree modeling. Reche et al. [29] presented another imagebased volumetric representation, in which a tree is represented by a set of grids with view-dependent textures, that are extracted from a few calibrated photographs. Following these previous works, our approach uses either image-based modeling or laser scanning to capture trees. Moreover, the recovered trees are not the modeling results but only as samples for the next analysis.

Textons and the Markov model. Our tree synthesis algorithm is inspired by texture synthesis algorithms. Texton [15], as a macro structure of an image, has been successfully used to synthesize large size textures $[6,17]$. A texture is a combination of textons and the distribution of textons is regarded as a Markov random field. The process of texture synthesis consists of three steps: defining textons, building the distribution graph of the textons, and finally planning an appropriate path in the distribution graph. The Markov model is widely used in speech recognition and synthesis [28], dynamical systems [32], video texture [11] and in motion texture [16]. For tree modeling, some biological research $[5,12]$ pointed out that the architecture of a plant relates to a Markov process.

\section{Tree model}

In this paper, we propose a two-level statistical model to depict certain botanical homogeneities of trees of the same species. Such botanical homogeneities are analyzed from tree samples, which are captured from the real world. We first give the mathematical representation of tree samples and then define our tree model.

\subsection{Sample representation}

In our method, the sample may be either a whole tree or a portion of a tree (Fig. 1(b)). A tree sample is represented by a pair consisting of an organ set $O$ and an organ connectivity $B$ (Fig. 1(c)). Corresponding to the tree structure in the real world, $O$ is a partially ordered set of organs and $B$ enumerates all directed connections between any two organs in $O$. In the tree sample, we assume that there are $M$ organs, namely $O=\left\{o_{1}, o_{2}, \ldots, o_{M}\right\}$, where $o_{1}$ is set as the root of the tree structure and for any $i<k, o_{k}$ cannot be the parent of $o_{i}$. Organ connectivity is defined by $B=\left\{b\left(o, o^{\prime}\right) \mid o \in O, o^{\prime} \in O\right.$, and $o^{\prime}$ grows from $\left.o\right\}$.

For each organ in $O$, a set of parameters $s$ is used to describe the organ's geometry. Similar to the previous 


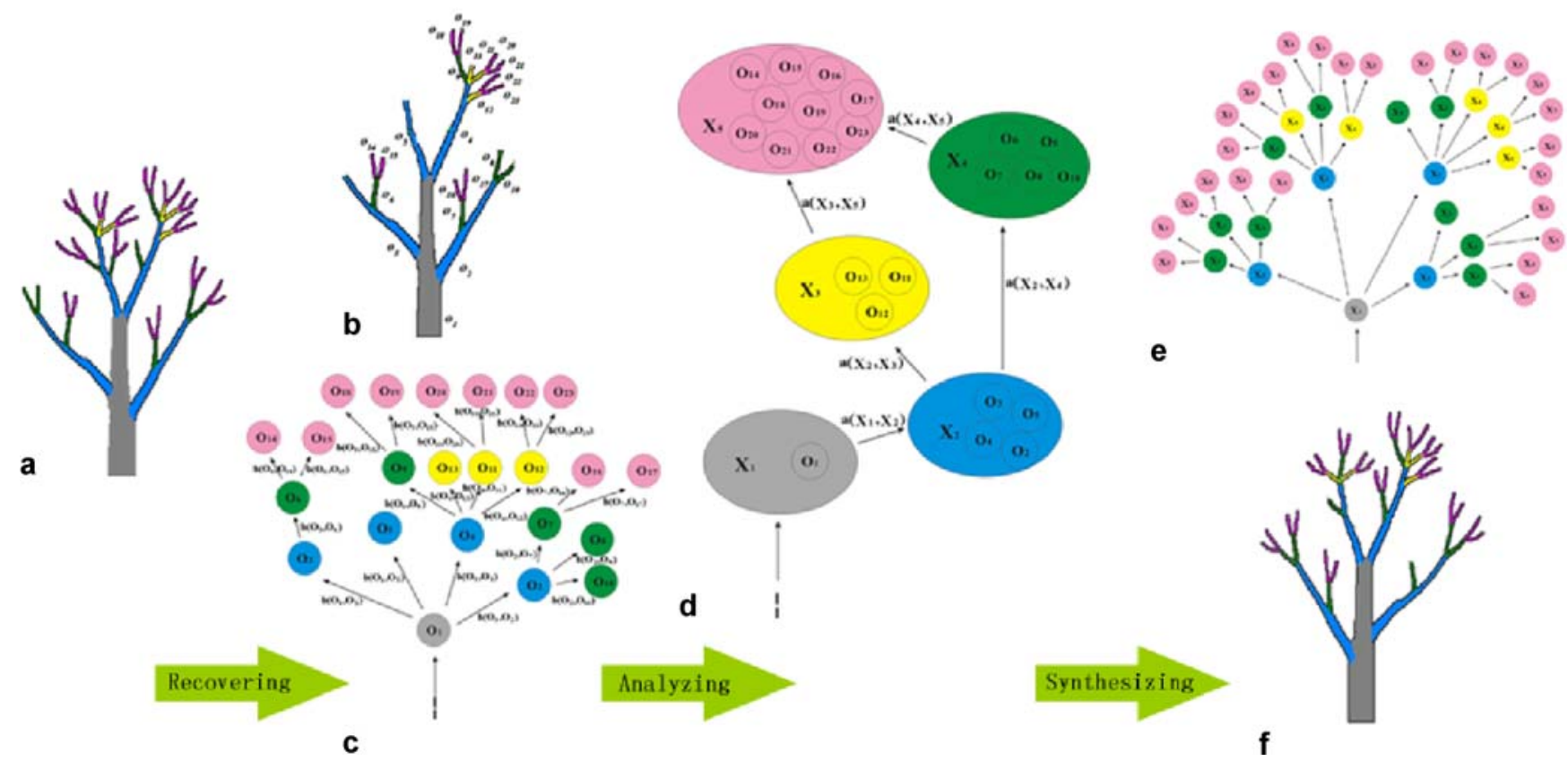

Fig. 1a-f. Overview of the algorithm. There are three steps in our tree modeling, recovering, analyzing and synthesizing. a A tree. Similar organs are marked in the same color. b Recovered tree sample, a portion of a real world tree. c The sample is represented by organ set $O=$ $\left\{o_{1}, o_{2}, \ldots, o_{M}\right\}$ and organ connectivity $B=\left\{b\left(o, o^{\prime}\right)\right\}$. d Two-level statistical model, the plantons $X$ and transitions of plantons $A$ learnt from tree sample after analyzing process. Similar organs (in the same color) are clustered in one planton. e A planton tree is synthesized from $\{X, A\}$ in the synthesizing process and each planton on such tree represents one organ. $\mathbf{f}$ The final synthesized new tree is similar to the original one but visually different

work $[13,38]$, our model defines three organ types such as the stem, leaf and flower. The geometry of a stem is represented by a central line (Bézier splines) with several circular cross-sections, while that of a leaf or a flower is represented by a polygon or a bounding box with textures, respectively (Fig. 2). These parameters used in the geometry representation are denoted by $s$. In the following, we use denotation $x . y$ to indicate that object $y$ is the member variable of object $x$, e.g. the parameter $s$ of organ $o$ is denoted by $o . s$. On the other hand, the denotation $v[k]$ is used to denote the $k$-th component of vector $v$.

To compare the similarity of two organs of the same type, we define a distance metric between two organs $o_{p}$ and $o_{q}$ as

$D\left(o_{p}, o_{q}\right)=\sum_{k} \alpha_{k}\left(o_{p} . s[k]-o_{q} . s[k]\right)^{2}$,

where $\alpha_{k}$ is the weight for the $k$-th component. The smaller the $D\left(o_{p}, o_{q}\right)$, the more the similar organs $o_{p}$ and $o_{q}$.

\subsection{A two-level statistical model}

At the low level of our two-level statistical model, a set of plantons is defined to depict similar organs statistically. At the high level, the transitions of plantons are used to describe the stochastic distribution of tree organs. Thus, a kind of tree can be represented by a planton set $X=\left\{x_{1}, x_{2}, \ldots, x_{N}\right\}$ together with the transitions of the plantons $A$ (a transition matrix) (Fig. 1(d)).

Each planton is defined by a triple $(\mathcal{L}, \mathcal{G}, \mathcal{N})$, where $\mathcal{L}$ is a cluster of organs with similar shape, $\mathscr{g}$ is the distribution of the organ's parameters in $\mathcal{L}$, and $\mathcal{N}$ is the distribution of the branch number of each organ in $\mathcal{L}$. In practice, we assume that $g$ is a high-dimensional Gaussian distribution and that $\mathcal{N}$ adopts a $1 D$ Gaussian distribution.

Under our assumption that the distribution of organs follows the first-order Semi-Markovian dynamics, the planton transition matrix $A$ can be used to represent the growing rule of a tree. Each element $a[i, j]$ in $A$, a transition, is also defined by a triple $(p, T, \Omega)$. The transition probability, $a[i, j] . p$, is the likelihood that an organ $o$ whose statistical properties are depicted by planton $x_{i}$ can branch another organ $o^{\prime}$ whose statistical properties are depicted by planton $x_{j}$.

$$
\alpha[i, j] . p=P\left(o^{\prime} \in x_{j} \cdot \mathcal{L} \mid o \in x_{i} \cdot \mathcal{L}\right) \quad b\left(o, o^{\prime}\right) \in B .
$$

Similarly, $a[i, j] . T$ and $a[i, j] . \Omega$ are, respectively, the distributions of the organ's location $t$ and the growing direction with respect to its parent organ. $t$ is normalized into the interval $[0,1]$ to represent the relative position. 


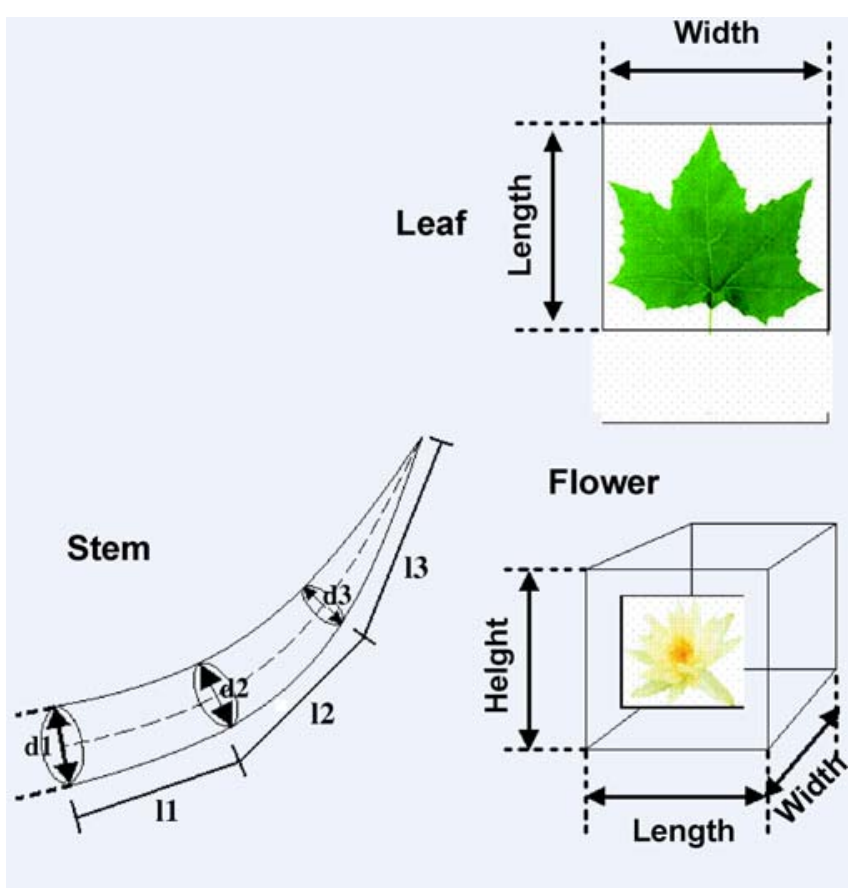

Fig. 2. Parameters of organs

The above model is equivalent to a directed graph in which each node represents a planton and the weight on each directed arc represents a transition. Similar models have been widely adopted in texture synthesis $[6,17]$, video texture [34] and motion textures [16]. In our approach, we further exploit it as the statistical model for tree modeling. Unlike conventional Markov models [4, 28], we use hybrid transitions, which depend not only on probabilities but also on other statistical features (location and direction distributions).

\subsection{Overview of our approach}

Our approach takes three steps to compose a new tree: (1) Acquiring tree samples from the real world. (2) Analyzing the tree samples to extract the statistical model, the plantons and the transitions of plantons. (3) Synthesizing new trees based on the learnt tree model (Fig. 1).

\section{Acquisition}

We use two methods to acquire samples from the real world. One is the laser scanning technique for capturing dense depth images of trees. Due to self-occlusion, it is difficult for this method to reconstruct foliage trees automatically. So, alternatively, the interactive image-based modeling technique is employed to reconstruct tree samples based on multiple photographs. In our method, as there is no need to reconstruct an entire tree and some elaborate tools are provided, the amount of user's interactions is acceptable and the time cost on recovering is relatively small. The recovering process is demonstrated in the electronic supplementary material (ESM).

\subsection{Data from range images}

A range camera is used to recover bare trees in winter. For each tree multiple photos of different views are shot. On each view, points with depths on 2D skeletons of stems are automatically extracted and then registered in a global coordinate (Fig. 3(a)). We take a two-step greedy algorithm to estimate parameters of stems from a registered skeleton points cloud. Firstly, these point clouds are sorted into a tree-structure, named skeleton-tree, by iteratively linking the closest points (Fig. 3(b)). Then, stems are extracted by fitting points on the skeleton-tree with Bézier curves. This process is conducted progressively, starting from the root, the first stem is created and updated while adjacent points are consequently taken into account. New stems will be created when the fitting error is larger than a threshold (Fig. 3(c)). Once the points for one stem is clustered, the stem radius is automatically computed from depth images (Fig. 3(d)). After these automatic steps, some interactions can be performed to adjust stems for better results.

\subsection{Data from photographs}

For foliage trees, we induct user interactions to guide the image-based tree recovering. For each type of organ, different interactive tools are developed to help the user to recover the shape of organs from calibrated photographs. For stems, a contour tracing method [22] is applied to extract their skeletons and radiuses. For leaves and flowers, tools are developed to extract organs' geometry and textures directly, the user only needs to locate their positions and growing directions on images.

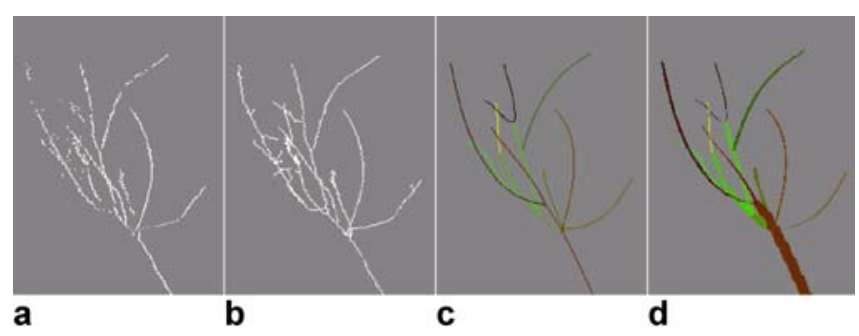

Fig. 3a-d. Fitting stems from skeleton points cloud. Only a part of a tree is shown as an example. a Registered skeleton points clouds. b Points are linked into a skeleton-tree c Points are fitted by Bézier curves and stems are extracted. d The stem's radius are automatically recovered from depth images along Bézier curves 


\section{Analyzing}

As the same type of organs can be found among all samples of the same species of trees, we conduct an analysis on multiple samples to extract the statistical tree model.

\subsection{Learning from a single sample}

Given a tree sample $(O, B)$, our approach learns the tree model parameters $(X, A)$ by finding a maximum likelihood solution.

$$
(\hat{X}, \hat{A})=\underset{(X, A)}{\arg \max } P(O, B \mid X, A))
$$

Considering $X$ and $A$ as hidden variables, we adopt the EM algorithm [3] to solve the maximum likelihood problem. All organs in the tree sample are classified by a k-mean algorithm according to the similarity distance metric defined in Sect. 3. In the first step (E-step), the distribution $(g, \mathcal{N})$ for each organ cluster and the transitions $(p, T, \Omega)$ among clusters are estimated, respectively. In the second step (M-step), according to the estimated distribution $(g, \mathcal{N})$ and transitions $(p, T, \Omega)$, all organs are reclassified and the clusters are updated. The E- and M-steps are executed repeatedly until the clusters remain unchanged.

\subsubsection{E-step}

Once the organ clusters $\left\{\mathscr{L}_{i} \mid i=1, \ldots, N\right\}$ are available, each organ cluster is initially regarded as a planton candidate, namely a planton candidate $\left\{x_{i}, x_{i} . \mathcal{L}=\mathscr{L}_{i}\right\}$. Thus, the shape distribution $g$ and the branching number distribution $\mathcal{N}$ of each candidate can be estimated by a maximum likelihood using Eq. 3,

$$
\begin{aligned}
x_{i} \cdot(\hat{g}, \hat{\mathcal{N}}) & =\underset{\left(x_{i} \cdot \mathscr{G}, x_{i} \cdot \mathcal{N}\right)}{\arg \max } P\left(x_{i} \cdot \mathcal{L} \mid x_{i} \cdot(\mathcal{g}, \mathcal{N})\right) \\
& =\arg \max \prod_{\text {all }} P\left(\text { o } x_{i} \cdot \mathcal{L}\right.
\end{aligned}
$$

Moreover, the element $a[i, j]$ in transition matrix $A$ is evaluated by the following formula

$\alpha[i, j] . p=\frac{\operatorname{count}\left\{b\left(o, o^{\prime}\right) \mid o^{\prime} \in \mathcal{L}_{i}, b\left(o, o^{\prime}\right) \in B\right\}}{\operatorname{count}\left\{b\left(o, o^{\prime}\right) \mid o^{\prime} \in O, b\left(o, o^{\prime}\right) \in B\right\}}$,

$a[i, j] . T$ and $a[i, j] . \Omega$ can be obtained in a way similar to the estimation of maximizing the likelihood of $g$ and $\mathcal{N}$.

\subsubsection{M-step}

When all planton candidates $X$ and the transition matrix $A$ are known, we need to re-classify all organs in organ set $O$ using the obtained parameters. Once given a classification label for organs $\left\{l_{1}, l_{2}, \ldots, l_{M}\right\}$, where $o_{k} \in x_{l_{k}} . \mathcal{L}$, according to Eq. 2, the likelihood that the sample is depicted by current classification can be evaluated as follows

$$
\begin{aligned}
P(O, B \mid X, A)= & P(O \mid X) \cdot P(B \mid A) \\
= & \prod_{k=1}^{M} P\left(o_{k} . s \mid x_{l_{k}} \cdot(g, \mathcal{N})\right. \\
& \cdot P\left(b\left(o_{p}, o_{k}\right) \mid \alpha\left[l_{p}, l_{k}\right](p, T, \Omega)\right) .
\end{aligned}
$$

where $o_{p}$ is the parent of $o_{k}$ in the sample. The first term is the probability that $o_{k}$ is depicted by the planton $x_{i}$, the second term reflects with how much possibility $o_{k}$ can grow from $o_{p}$ under the current planton transitions. Therefore, the optimal re-classification of organs can be derived by maximizing $P(O, B \mid X, A)$.

\section{Algorithm 1 M-step for classification}

1. Initialization

$$
H_{1}(i)=P\left(o_{1} . s \mid x_{i}(g, \mathcal{N})\right)
$$

2. For $k=2$ to $M$ ( $M$ is the number of organs)

$$
\begin{array}{r}
H_{k}(i)=\max _{j}\left[H_{k-1}(j) * P\left(o_{k} . s \mid x_{i}(g, \mathcal{N})\right)\right. \\
\cdot P(b(j, i) \mid a[j, i](p, T, \Omega))] \\
I_{k}(i)=\underset{j}{\arg \max }\left[H_{k-1}(j) * P\left(o_{k} . s \mid x_{i}(g, \mathcal{N})\right)\right. \\
\cdot P(b(j, i) \mid a[j, i](p, T, \Omega))]
\end{array}
$$

3. Final solution

$$
\begin{aligned}
& H=\max _{i} H_{M}(i) \\
& I_{M}=\underset{i}{\arg \max } H_{M}(i)
\end{aligned}
$$

4. Backtrack the label $l_{k}$

For $k=M-1$ to 1

$$
l_{M}=I_{M}, l_{k}=I_{k+1}\left(l_{k+1}\right)
$$

All labels $\left\{l_{k}\right\}$ indicate which planton $o_{k}$ belongs to.

5. Refine the clusters

Evaluate error $e_{k}$ in $l_{k}$-th planton

If $\left(e_{i}>e_{\max }\right)$

Create a new cluster and insert $o_{k}$ into it Else

Insert $o_{k}$ into cluster $\mathscr{L}_{l_{k}}$

A dynamic programming algorithm is used to find the optimal classification label $\left\{l_{1}, l_{2}, \ldots, l_{M}\right\}$. It can be considered as an iterative searching process from the root organ $o_{1}$ to the current organ $o_{k}$ to find the classification of $o_{1}, \ldots, o_{k}$ to maximize $P(O, B \mid X, A)$. When $k$ reaches $M$, the classification of $o_{1}, \ldots, o_{M}$ with the maximum probability is the desirable solution. We use $H_{k}(i)$ to represent the maximum likelihood of all classifications of $o_{1}, \ldots, o_{k}$, where the $k$-th organ belongs to the $i$-th planton. $I_{k}(i)$ is used to represent the label of the planton 
to achieve $H_{k}(i)$. The algorithm is summarized in Algorithm 1. In the algorithm, we use a threshold $e_{\max }$ to control whether the statistical properties of an organ can be depicted by a planton. It affects the number of resultant plantons. The greater the $e_{\max }$ is, the fewer the resultant plantons are.

\subsection{Learning from multi-samples}

Given $K$ samples $\left(O_{1}, B_{1}\right), \ldots,\left(O_{K}, B_{K}\right)$, Eq. 2 can be extended to

$$
\begin{aligned}
(\hat{X}, \hat{A}) & =\underset{(X, A)}{\arg \max } P\left(\left(O_{1}, B_{1}\right), \ldots,\left(O_{K}, B_{K}\right) \mid X, A\right) \\
& =\underset{(X, A)}{\arg \max } \prod_{i=1}^{K} P\left(\left(O_{i}, B_{i}\right) \mid X, A\right) .
\end{aligned}
$$

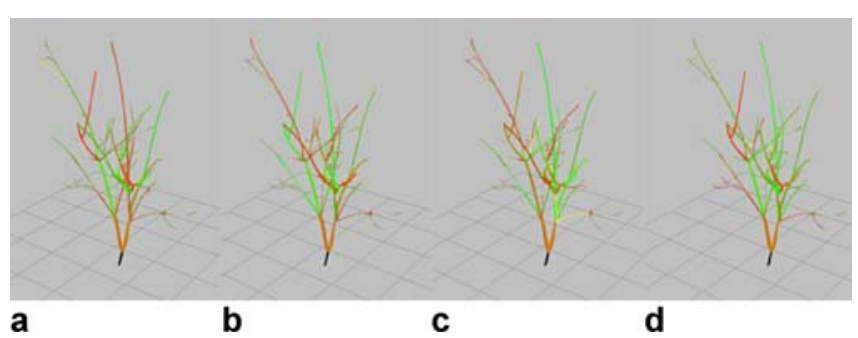

Fig. 4a-d. Classifications of stems in the EM algorithm. a An initial classification of 25 plantons. $\mathbf{b}$ and $\mathbf{c}$ Intermediate classifications of 23 plantons and 20 plantons, respectively. $\mathbf{d}$ is the final classification of 20 planton

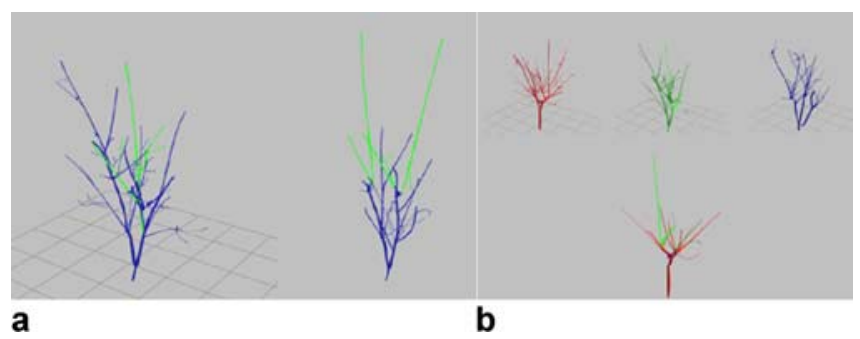

Fig. 5a,b. Synthesizing a new tree. a A step of the synthesis from one sample (left) to a new tree (right). Some organs are highlighted to outline the correspondences between the new tree and the sample. b A step of the synthesis from three samples (upper) to a new tree (bottom). Organs are colored differently in order to indicate from which sample they are synthesized

Table 1. Data of recovering and analyzing tree samples from range images

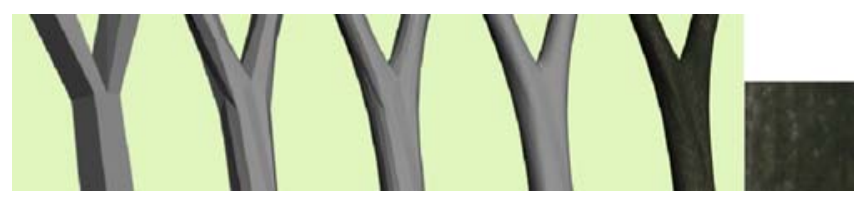

Fig. 6. Mesh subdivision and texture synthesis

The solution for Eq. 6 is similar to Eq. 2 except for a few minor modifications. At each E-step, $X$ and $A$ are estimated from all organs in $\cup_{i=1}^{K} O_{i}$. At each M-step, the classification is derived by maximizing Eq. 5 for each sample $\left(O_{i}, B_{i}\right)$.

\section{Synthesizing}

With the learnt plantons and transition matrix, it is efficient to synthesize many new statistically similar trees by randomly sampling from $\{X, A\}$. Since our tree model distinguishes the high-level organ distribution (transition matrix $A$ ) from the low-level distribution (plantons $X$ ), a two-step approach to synthesize new trees is formed. A tree-structure, namely a planton tree, which represents the biological branching structures of a novel tree, is first generated. With the planton tree, organs are then generated for each planton.

\subsection{Generating a planton tree}

We use a recursive algorithm to synthesize planton trees. It starts at the root planton in the planton set $X$. For the current planton $x_{i}$, the number of its child planton numbers is specified by $x_{i} \cdot \mathcal{N}$, and each child planton is randomly chosen from the transition matrix; both its position and growing direction are also randomly estimated according to $x_{i} . T$ and $x_{i} . \Omega$. The algorithm is terminated when no new planton can be visited (Fig. 1(e)).

\subsection{Generating organs}

By traversing the planton tree, a new tree is created. We synthesize one organ at each planton. For each planton $x$, the parameters of a new organ are evaluated according to the distribution $x . g$, and an organ $o$ is selected from the

\begin{tabular}{llllll}
\hline & $\begin{array}{l}\text { Skeleton } \\
\text { points }\end{array}$ & $\begin{array}{l}\text { Initial } \\
\text { stems }\end{array}$ & $\begin{array}{l}\text { Pruned } \\
\text { stems }\end{array}$ & $\begin{array}{l}\text { Plantons } \\
\text { after } \\
\text { k-mean }\end{array}$ & $\begin{array}{l}\text { Plantons } \\
\text { after EM } \\
\text { algorithm }\end{array}$ \\
\hline Fig. 8(a) & 6719 & 623 & 187 & 25 & 22 \\
Fig. 8(b) & 5724 & 654 & 141 & 25 & 20 \\
Fig. 8(c) & 6409 & 608 & 195 & 26 & 22 \\
Fig. 8(a,b, c) & - & - & - & 45 & 32 \\
\hline
\end{tabular}


Table 2. Data of recovering and analyzing tree samples from photographs

\begin{tabular}{llll}
\hline & $\begin{array}{l}\text { Input } \\
\text { organs }\end{array}$ & $\begin{array}{l}\text { Plantons } \\
\text { after } \\
\text { k-mean }\end{array}$ & $\begin{array}{l}\text { Plantons } \\
\text { after EM } \\
\text { algorithm }\end{array}$ \\
\hline Fig. 8(d) & 65 & 15 & 15 \\
Fig. 8(e) & 50 & 13 & 13 \\
Fig. 8(f) & 53 & 13 & 14 \\
Fig. 8(d, e, f) & - & 24 & 22 \\
\hline
\end{tabular}

organ cluster $x . \mathcal{L}$ such that the organ's parameter o.s best fits the derived parameters. Finally, the geometry of the selected organ is scaled to the appropriate size and is located at the junction between its parent organ and itself (Fig. 1(f)).

\section{Implementation and results}

\subsection{Results of recovering}

We used the DeltaSphere ${ }^{T M}-3000$ 3D scene digitizer to scan trees in the real world. For each tree, two to four views were shot. Due to the different complexity of the trees, extracting skeletons, registering skeleton points, building a skeleton tree and automatically recovering stems took about five to ten minutes and an extra two to three hours were spent on interactively adjusting the connections of stems on an Intel Pentium IV 2.4GHz computer with $1 \mathrm{~GB}$ memory. Table 1 shows the statistics data regarding the recovering of 3D samples shown in Fig. 8(a), (b) and (c). In our experiments, it took a total of 4 or 5 hours to recover organs from photographs for one sample. Some recovered results are shown in Fig. 7 and more

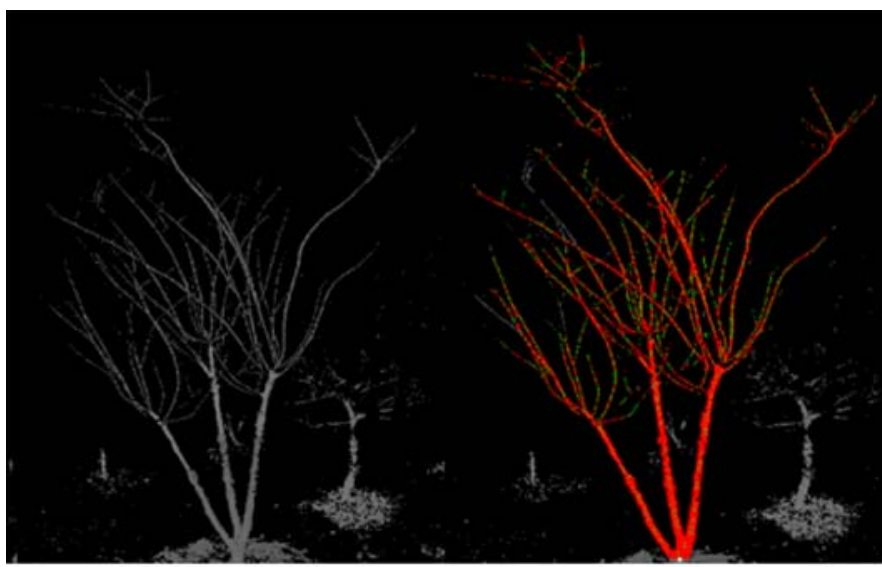

a b

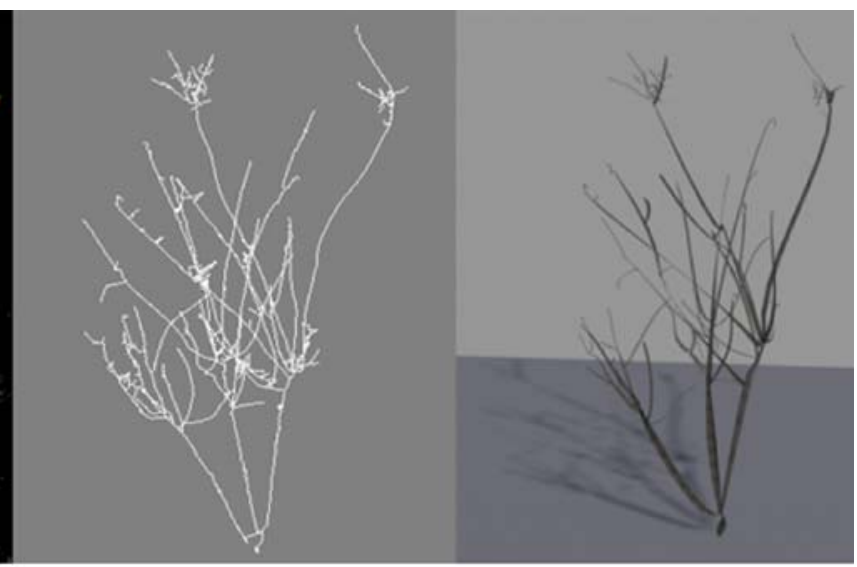

C d

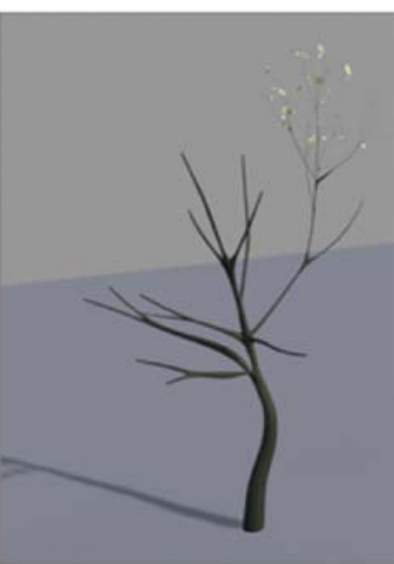

h

Fig. 7a-h. Procedure of recovering 3D tree samples from range images and photographs. a (a) One of captured depth images. b Range points (in red). c The skeleton-tree in the global coordinate system. $\mathbf{d}$ The final recovered result. e One of the photographs. $\mathbf{f}$ The input skeleton by the user. $\mathbf{g}$ The registered skeleton. $\mathbf{h}$ The recovered result. More recovering details are shown in recovering.avi 


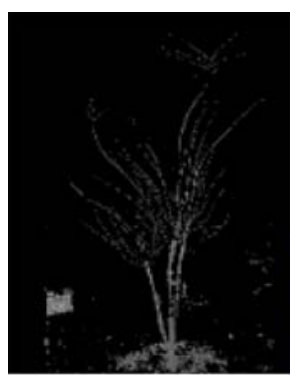

a

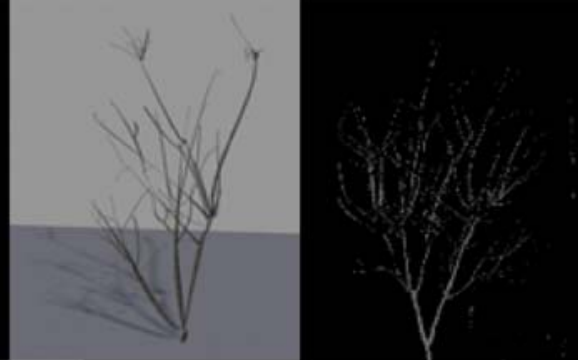

b

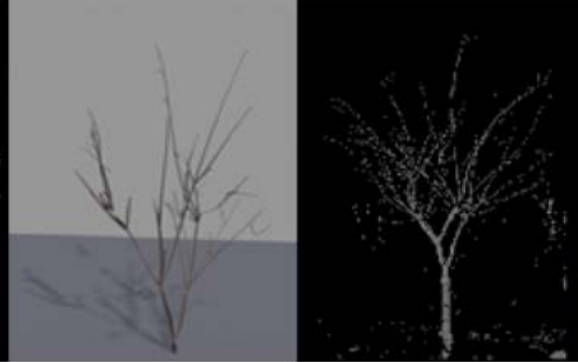

c

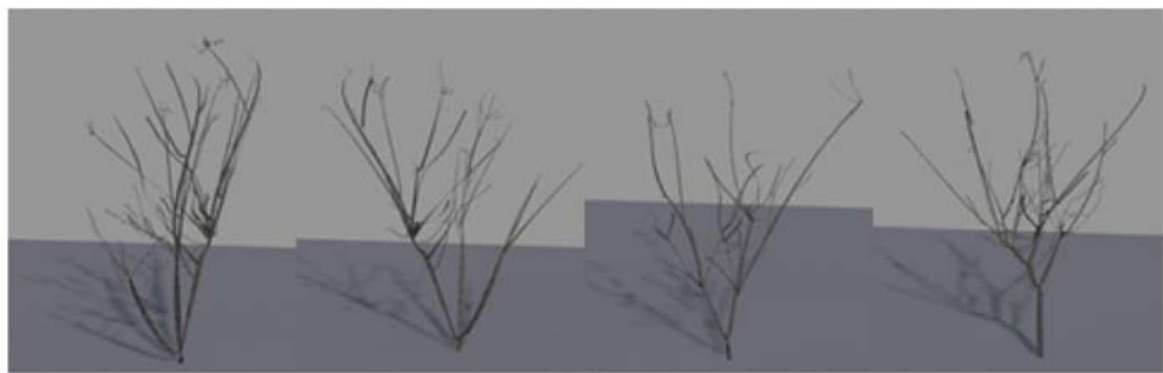

d

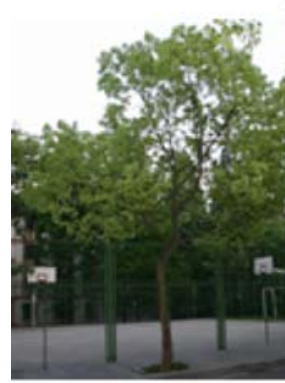

e

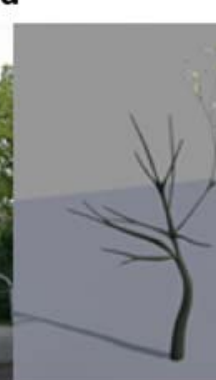

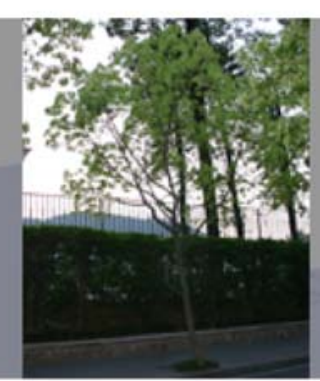

f
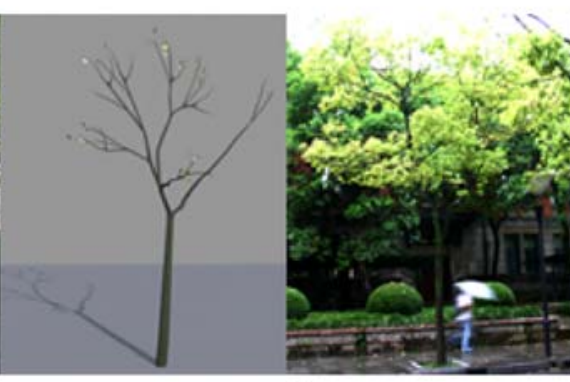

g

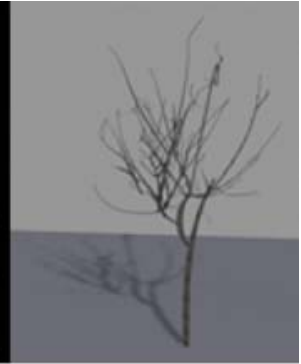

列

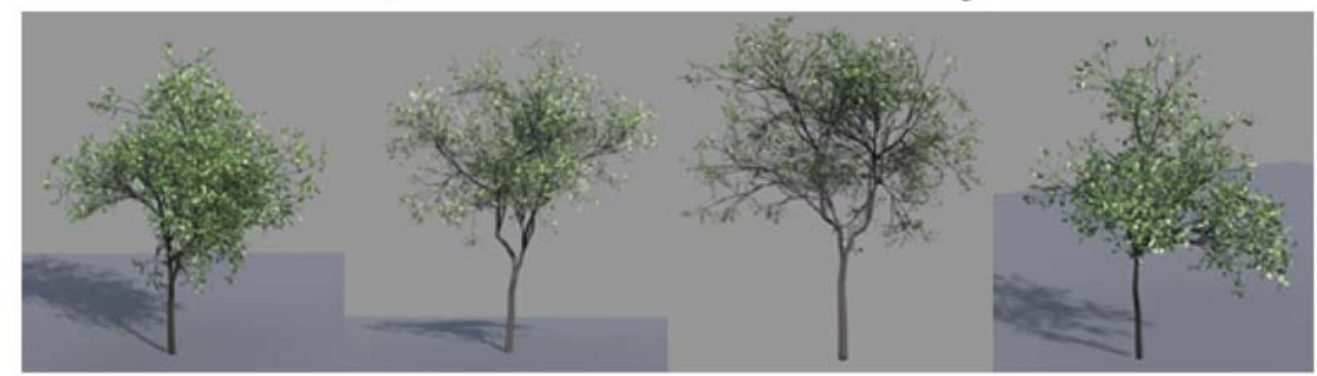

h

Fig. 8a-h. Synthesized results. a, b and $\mathbf{c}$ The range images and the respective recovered 3D samples. d The synthesized new tree. (e)', f and $\mathbf{g}$ Photographs and the recovered 3D samples. $\mathbf{h}$ The synthesized results

details of the recovering process are demonstrated in the ESM. Note that the repetition of leaves and flowers in a tree is very high. Therefore, compared with stems, fewer leaves and flowers are recovered. 3D tree samples recovered by image-modeling technique are shown in Fig. 8(e), (f) and (g) (one of photographs is shown on the left-hand side and the sample is shown on the right-hand side) and Fig. 9(b) and (f).

\subsection{Results of learning}

Our learning algorithm iteratively clusters organs from given samples. The initial classification is obtained by using a k-mean clustering algorithm. The numbers of learnt plantons are listed in Tables 1 and 2. In both Tables 1 and 2 , the last row is the number of learnt plantons from three samples. Figure 4 illustrates a few steps of it- 

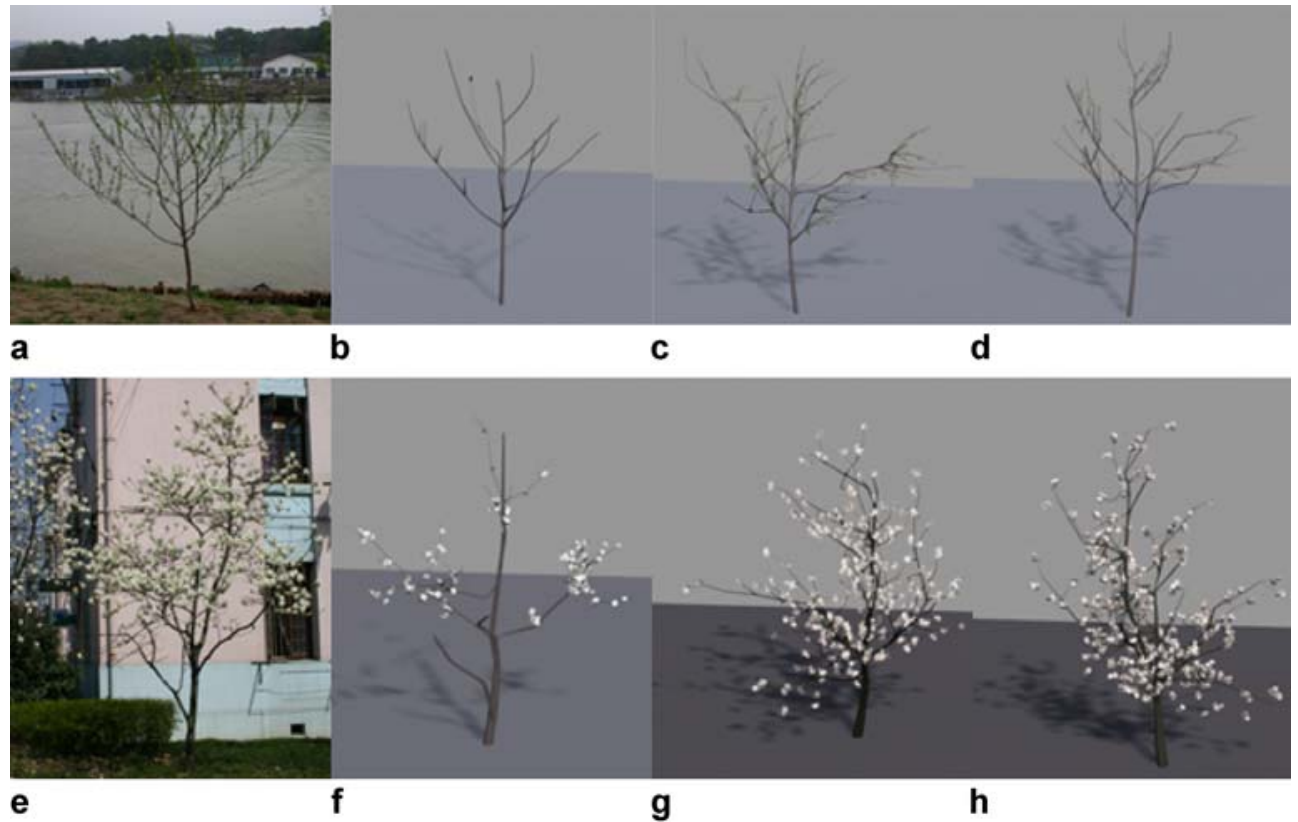

Fig. 9a-h. More synthesized results. a and e Captured photographs. b and $\mathbf{f}$ The recovered 3D samples. $\mathbf{c}, \mathbf{d}, \mathbf{g}$ and $\mathbf{h}$ The synthesized results

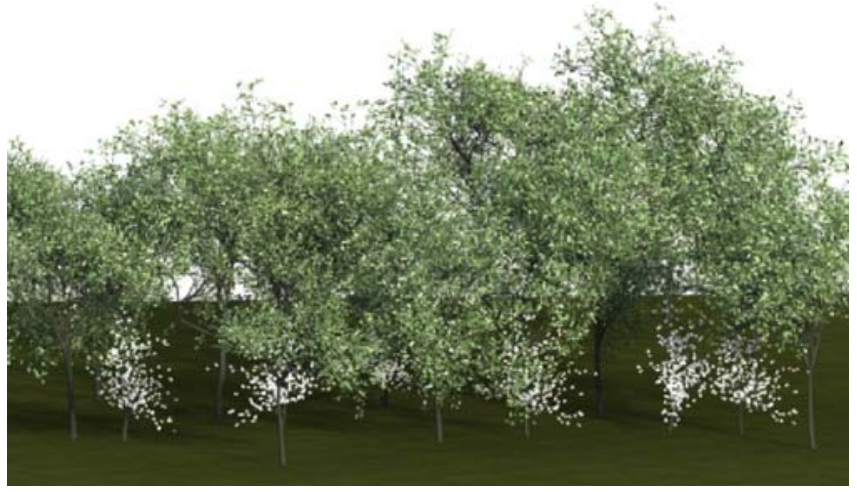

Fig. 10. Trees on a timberland

erations in the stem classification. Stems clustered in the same planton are shown in the same color. The variation of colors of the same organ in Fig. 4(a d) reflects the result of different classifications. The ESM, demonstrates the complete classification process of Fig. 4.

\subsection{Results of synthesis}

Some steps in synthesis are shown in Fig. 5 and the complete process of synthesis is demonstrated in the ESM. Synthesized parametric organs are converted into meshes and exported to the software 3D Studio Max for final rendering. For stems, a generalized mesh subdivision algorithm [20] is used to achieve natural transitions at the branching locations. Note that textures of the stems are difficult to recover completely from the captured images; we adopt a texture synthesis method to generate textures on meshes [39] based on the recovered texture sample. Figure 6 shows several intermediate meshes in the subdivision and the final textured mesh. Different from the case of stems, the textures for leaves or flowers are directly extracted from images. When rendering leaves, textured polygons are used. For flowers, only the textured bounding boxes are rendered.

Figure 8 illustrates the synthesized results with one tree sample or multiple samples. Trees shown in Fig. 8(d) are synthesized from samples in Fig. 8(a), (b) and (c) and trees in Fig. 8(h) are synthesized based on Fig. 8(e),(f) and (g). Further results of recovering and synthesizing by our method are shown in Fig. 9. Moreover, a total of 15 and 21 trees of three kinds are synthesized and positioned in the scenes of Fig. 10 and Fig. 11, demonstrating the potential of our method for many computer graphic applications.

\section{Conclusion and discussion}

In this paper, we propose an automatic analyzing and synthesizing approach for tree modeling. Instead of using external rules and cumbersome parameters, our approach models realistic trees from samples. Automatic algorithms, as well as several interactive tools, are developed to reconstruct tree samples based on either range images or photographs. We use a two-level statistical model to 


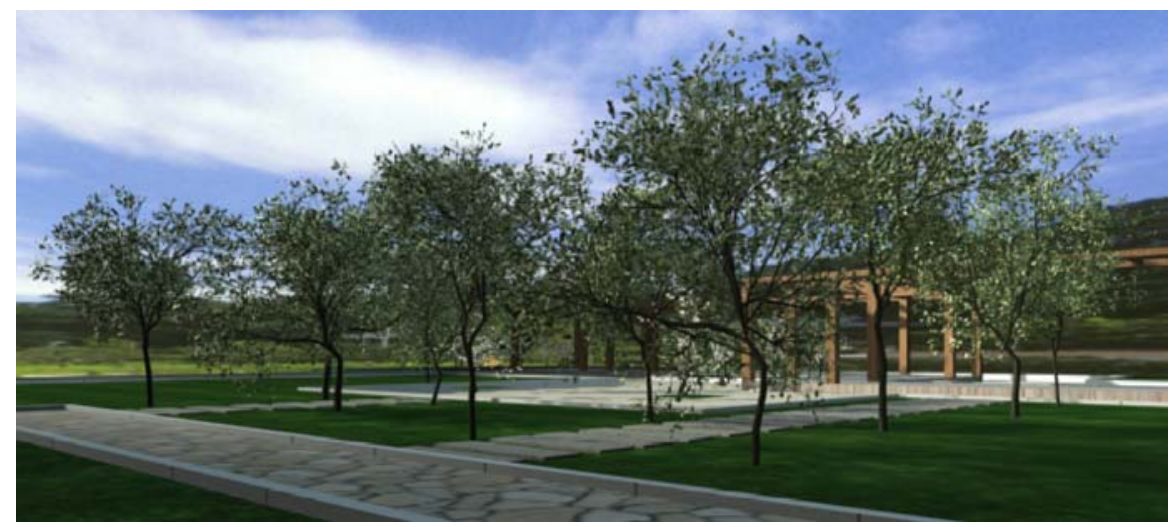

Fig. 11. Trees in a garden

represent trees. The maximum likelihood estimation is applied to extract the statistical properties of trees. With our two-level model, similar but visually different trees of the same species trees can be synthesized efficiently.

Compared with traditional tree modeling methods, our approach does not require the user to have much biological knowledge and modeling experience. Compared with many image-based plant modeling approaches, our approach is capable of synthesizing new trees efficiently. Considering the complex structure of trees and the limitations of the acquisition techniques, another advantage of our method is that only a portion of a real world tree needed to be recovered, which greatly reduces the cost for capturing trees and obtaining sound synthesized results.

Nevertheless, there are also some limitations of our method due to its statistical feature. As plantons and planton transitions are derived from repetitive organs, if the samples of organs is insufficient, the synthesized results lack variety or fidelity. In our implementation, we use several thresholds to control the recovering and analyzing process to obtain sufficient organs and find enough plantons. We define the minimum organ number $M_{\min }$ in a planton to evaluate whether the statistical distribution of the organ is captured, the maximum threshold $e_{\max }$ is adopted to classify organs into the plantons, and the minimum eligible planton number $N_{\min }$ is used to ensure the statistical properties of the planton distribution. Experimental results show that more than three organs in an eligible planton and more than eight eligible plantons are capable of synthesizing the realistic trees in Fig. 8(h).

We assume that the transitions of plantons conform to the first-order semi-Markov dynamics, because the first-order model is enough to reflect the statistical properties of trees and is easy to be learned. However, from a biological point of view, it is as yet a simplified assumption. In the future, more elaborate models should be chosen and more work should be done on the statistical representation of trees to produce more complex results. Furthermore, it would be useful to develop a controllable synthesis algorithm so that users can easily design trees to meet their particular requirements.

Acknowledgement This work is supported by the National Natural Science Foundation of China (Grant No.60203014, 60021201), the 973 Program of China (Grant No.2002CB3121 01), and the Cultivation Fund of the Key Scientific and Technical Innovation Project, Ministry of Education of China (No. 705027).

\section{References}

1. Aono, M., Kunii, T.L.: Botanical tree image generation. IEEE Comput. Graph. Applic. 4(5), 10-34 (1984)

2. Boudon, F., Prusinkiewicz, P., Federl, P., Godin, C., Karwowski, R.: Interactive design of bonsai tree models. Comput. Graph. Forum 22(3), 591-599 (2003)

3. Dempster, N.M., Laird, A.P., Rubind, B.: Maximum likelihood from incomplete data via the EM algorithm. J. R. Statist. Soc. B 39, 185-197 (1977)

4. Drake, A.W.: Observation of a Markov process through a noisy channel. $\mathrm{PhD}$ thesis, Massachusetts Institute of Technology (1962)
5. Durand, J.B., Guédon, Y., Caraglio, Y.: Analysis of the plant architecture via tree-structured statistical models: the hidden Markov trees. In: Proceedings of the Fourth International Workshop on Functional-Structural Plant Models, pp. 61-64, Montpellier, France (2004)

6. Efros, A. A., Freeman, W. T.: Image quilting for texture synthesis and transfer. In: Proceedings of the 28th Annual Conference on Computer Graphics and Interactive Techniques, pp. 341-346 (2001)

7. Fournier, A., Fussel, D., Carpenter, L.: Computer rendering of stochastic models. Communic. ACM 25(6), 371-384 (1982)
8. Fournier, A., Grindal, D.A.: The stochastic modeling of trees. In: Proceedings of Graphics Interface'86, pp. 164-172 (1986)

9. Galbraith, C., Muendermann, L., Wyvill, B.: Implicit visualization and inverse modeling of growing trees. Comput. Graph. Forum 23(3), 351-360 (2004)

10. Galbraith, C., Muendermann, L., Wyvill, B.: Blob Tree Trees. Proceedings of Computer Graphics International Conference, CGI, pp. 78-85, 2004

11. Greene, N.: Voxel space automata: modeling with stochastic growth processes in voxel space. (In: Proceedings of Siggraph 89) Comput. Graph. 23(4), 175-184 (1989) 
12. Guédon, Y., Barthélémy, D., Caraglio, Y., Costes E.: Pattern analysis in branching and axillary flowering sequences. J. Theoret. Biol. 212, 481-520 (2001)

13. Holton, M.: Strands, gravity, and botanical tree imagery. Comput. Graph. Forum 13(1), 57-67 (1994)

14. Honda, H.: Description of the form of trees by the parameters of the tree-like body: effects of the branching angle and the branch length on the shape of the tree-like body. J. Theoret. Biol. 31, 331-338 (1971)

15. Julesz, B.: Textons, the elements of texture perception and their interactions. Nature 290, 91-97 (1981)

16. Li, Y., Wang, T., Shum, H.: Motion texture: a two-level statistical model for character motion synthesis. ACM Trans. Graph. 21(3), 465-472 (2002)

17. Liang, L., Liu, C., Xu, Y., Guo, B., SHUM, H.: Real-time texture synthesis by patch-based sampling. ACM Trans. Graph. 20(3), 127-150 (2001)

18. Lintermann, B., Deussen, O.: Interactive modeling of plants. IEEE Comput. Graph. Applic. 19(1), 56-65 (1999)

19. Lindenmayer, A.: Mathematical models for cellular interaction in development. Parts I and II. J. Theoret. Biol. 18, 280-315 (1968)

20. Loop, C.: Smooth subdivision surfaces based on triangles. Master's thesis, University of Utah, Department of Mathematics (1987)

21. Mandelbrot, B.B.: The Fractal Geometry of Nature. Freeman and Co., San Francisco, CA (1982)

22. Mortensen, E.N., Barret, W.A.: Intelligent scissors for image composition. In:
SIGGRAPH'1995, pp. 191-199 (1995)

23. Oppenheimer, P.E.: Real-time design and animation of fractal plants and trees. (SIGGRAPH 86 Conference Proceedings) Comput. Graph. 20, 55-64 (1986)

24. Prusinkiewicz, P., Hammel, M., Mjolsness, E.: Animation of plant development. In: SIGGRAPH 93 Conference Proceedings, pp. 351-360, August (1993)

25. Prusinkiewicz, P., James, M., Mech, R.: Synthetic topiary. In: Proceedings of SIGGRAPH, pp. 351-358. ACM SIGGRAPH, New York (1994)

26. Prusinkiewicz, P., Lindenmayer, A.: The Algorithmic Beauty of Plants. Springer, Berlin Heidelberg New York (1996)

27. Prusinkiewicz, P., Mundermann, L., Karwowski, R., Lane, B.: The use of positional information in the modeling of plants. In: Proceeding of SIGGRAPH 2001, pp. 289-300 (2001)

28. Rabiner, L.: A tutorial on hidden Markov models and selected applications in speech recognition. Proc. IEEE 77(2), 257-285 (1989)

29. Reche, A., Martin, I., Drettakis, G.: Volumetric reconstruction and interactive rendering of trees from photographs. (SIGGRAPH Conference Proceedings) ACM Trans. Graph. 23(3), 720-727 (2004)

30. Reeves, W.T.: Particle systems-a technique for modeling a class of fuzzy objects. ACM Trans. Graph. 2(2), 91-108 (1983)

31. Reffye, D.P., Edelin, C., Francon, J., Jaeger, M., Puech, C.: Plant model faithful to botanical structure and development. SIGGRAPH Comput. Graph. 22, 151-158 (1988)
32. Reissell, L.M., Dinesh K.P.: Modeling stochastic dynamical systems for interactive simulation. Comput. Graph. Forum 20(3), 339-348 (2001)

33. Sakaguchi, T., Ohya, J.: Modeling and animation of botanical trees for interactive virtual environments. In: Proceedings of the ACM Symposium on Virtual Reality Software and Technology, pp. 139-146, London (1999)

34. Schodl, A., Szeliski, R., Salesin, D.H., Essa, I.: Video textures. In: Proceedings of ACM SIGGRAPH 2000, pp. 489-498 (2000)

35. Shlyakhter, I., Rozenoer, M., Dorsey, J., Teller, S.: Reconstructing 3D tree models from instrumented photographs. Comput. Graph. Appl. 21(3), 53-61 (2001)

36. Ulam, S.M.: On some mathematical problems connected with patterns of growth of figures. In: Proceedings of the Symposia in Applied Mathematics, vol. 14, pp. 215-224, Am. Math. Soc., Providence, RI (1962)

37. Viennot, X.G., Eyrolles, G., Janey, N., Arques, D.: Combinatorial analysis of ramified patterns and computer imagery of trees. In: Computer Graphics (SIGGRAPH 89 Conference Proceedings), vol. 23, pp. 31-40 (1989)

38. Weber, J., Penn, J.: Creation and rendering of realistic trees. In: SIGGRAPH 1995, pp. 119-128 (1995)

39. Zhang, J., Zhou, K., Velho, L., Guo, B., Shum, H.: Synthesis of progressively-variant textures on arbitrary surfaces. ACM Trans. Graph. 22(3), 295-302 (2003)
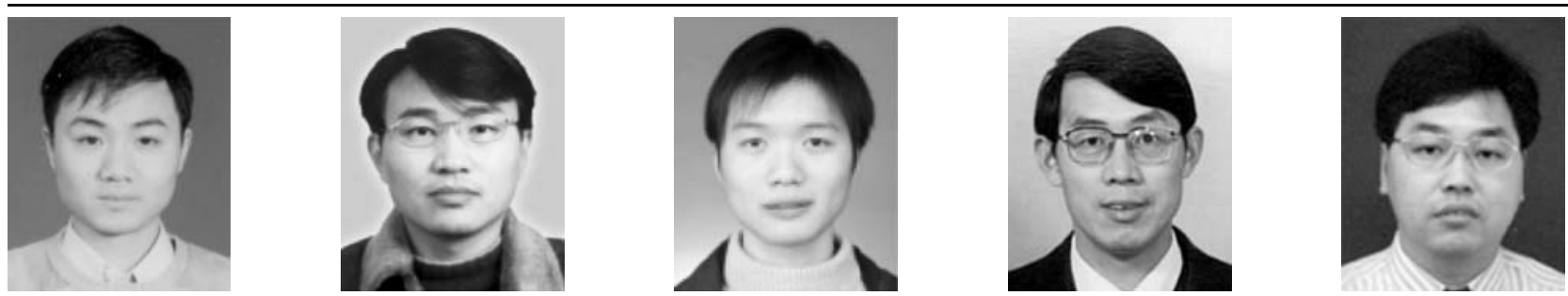

RUI WANG received the BSc degree in Computer Science from Zhejiang University in 2001. $\mathrm{He}$ is currently a $\mathrm{PhD}$ candidate in the State Key Laboratory of CAD \& CG of Zhejiang University. His research interests are image-based modeling, machine learning and geometry approximation.

Wei HuA received the PhD degree in Applied Mathematics from Zhejiang University in 2002. $\mathrm{He}$ is currently an associate professor of the State Key Laboratory of CAD \& CG of Zhejiang University. His research interests include realtime simulation and rendering, virtual reality and software engineering.
ZILONG DONG received the BSc degree in Computer Science from Zhejiang University in 2004. He is currently a PhD candidate in the State Key Laboratory of CAD \& CG of Zhejiang University. His research interests are image processing and machine learning.

Qunsheng Peng is Professor of Computer Graphics at Zhejiang University. His research interests include realistic image synthesis, computer animation, scientific data visualization, virtual reality, bio-molecule modeling. Prof. Peng graduated from Beijing Mechanical College in 1970 and received a Ph.D from the Department of Computing Studies, University of East Anglia in 1983. He is currently serving as a member of the editorial boards of several international and Chinese journals.

HUJUN BAO received his Bachelor and $\mathrm{PhD}$ degrees in Applied Mathematics from Zhejiang University in 1987 and 1993 . His research interests include modeling and rendering techniques for large scale of virtual environments and their applications. He is currently the director of State Key Laboratory of CAD \& CG of Zhejiang University. $\mathrm{He}$ is also the principal investigator of a virtual reality project sponsored by Ministry of Science and Technology of China. 